\title{
Three-terminal transport through a quantum dot in the Kondo regime: Conductance, dephasing and current-current correlations
}

\author{
David Sánchez and Rosa López \\ Département de Physique Théorique, Université de Genève, CH-1211 Genève 4, Switzerland
}

(Dated: June 19, 2018)

\begin{abstract}
We investigate the nonequilibrium transport properties of a three-terminal quantum dot in the strongly interacting limit. At low temperatures, a Kondo resonance arises from the antiferromagnetic coupling between the localized electron in the quantum dot and the conduction electrons in source and drain leads. It is known that the local density of states is accessible through the differential conductance measured at the (weakly coupled) third lead. Here, we consider the multiterminal current-current correlations (shot noise and cross correlations measured at two different terminals). We discuss the dependence of the current correlations on a number of external parameters: bias voltage, magnetic field and magnetization of the leads. When the Kondo resonance is split by fixing the voltage bias between two leads, the shot noise shows a nontrivial dependence on the voltage applied to the third lead. We show that the cross correlations of the current are more sensitive than the conductance to the appearance of an external magnetic field. When the leads are ferromagnetic and their magnetizations point along opposite directions, we find a reduction of the cross correlations. Moreover, we report on the effect of dephasing in the Kondo state for a two-terminal geometry when the third lead plays the role of a fictitious voltage probe.
\end{abstract}

PACS numbers: 72.15.Qm, 72.70.+m, 73.63.Kv

\section{INTRODUCTION}

The Kondo effect represents a distinguished example of strong many-body correlations in condensed matter physics $\frac{1}{=}$ Over the last fifteen years, much effort has been made in understanding the implications of the Kondo effect on the scattering properties of phase-coherent conductors. Indeed, the electric transport through a quantum dot connected to two terminals becomes highly correlated when the temperature $T$ is lowered below a characteristic energy scale given by $k_{B} T_{K}{ }^{2}$ At equilibrium, the Kondo temperature $T_{K}$ depends on the parameters of the system, i.e., the coupling of the dot to the external leads due to tunneling, the dot onsite repulsion (charging energy) and the position of the resonant level relative to the Fermi energy $E_{F}$. All of them can be tuned in a controlled way $\underline{\underline{3}}$

In a quantum dot with a sufficiently large charging en$\operatorname{ergy}\left(U \gg k_{B} T\right)$ and a single energy level well below $E_{F}$, the dynamics of the quasilocalized electron becomes almost frozen. Therefore, a quantum dot can be viewed as an artificial realization (at the nanoscale) of a magnetic impurity with spin $S=1 / 2$. At very low temperatures $\left(T<T_{K}\right)$, charge fluctuations in the dot are suppressed and there arises an effective antiferromagnetic interaction between the electrons of the reservoir and the $S=1 / 2$ localized moment. Remarkably, the measured conductance reaches the maximum value for a quantum channel $\left(2 e^{2} / h\right)$ and the dot appears to be perfectly transparent when a small voltage $e V_{\text {sd }}$ is applied between the source and the drain contacts. Nevertheless, the coherent correlated motion of the delocalized electrons forming the Kondo cloud can be disturbed when either the bias voltage or the temperature are of the order of $T_{K}$. In such a case, the many-body wave function of the Kondo state is expected to suffer from dephasing, leading to a decrease in the conductance. This issue has recently attracted a lot of attention 4.5.6. In this work, we mimic in a phenomenological way the effect of dephasing on the transport properties of a two-terminal quantum dot in the Kondo regime by introducing a fictitious voltage probe.

Now, in the absence of dephasing, the building block of the Kondo resonance is a narrow peak (of width $\sim k_{B} T_{K}$ ) around $E_{F}$ in the local density of states (LDOS) of the dot. However, full quantum-dot spectroscopy of the LDOS cannot be accomplished with a two-terminal transport setup. In particular, one cannot gain experimental access to the predicted large voltage induced splitting of the LDOS when $e V_{\mathrm{sd}}>k_{B} T_{K}{ }^{7.8 .9 .10} \mathrm{~A}$ way to circumvent this problem is by attaching a third lead, as demonstrated independently by Sun and Guo 11 and Lebanon and Schiller ${ }^{12}$ In subsequent laboratory work, De Franceschi et al $\stackrel{13}{n}$ observed a split Kondo resonance by employing a slightly modified technique - one of the leads was replaced by a narrow wire driven out of equilibrium where left and right moving carriers have different electrochemical potentials.

Motivated in part by the works cited in the preceding paragraph, we are concerned in this paper as well with the nonequilibrium Kondo physics and the fluctuations of the current through a quantum dot attached to three leads. As is well known, the investigation of the currentcurrent correlations in mesoscopic conductors has been a fruitful area of research ${ }^{14}$ Nevertheless, there are still very scarce applications to strongly correlated systems as the shot noise is a purely nonequilibrium property, and thus more difficult to treat. Hershfield ${ }^{15}$ calculates the zero-frequency shot noise using perturbation theory in the charging energy (valid when the Kondo correlations are not large; e.g., at $T>T_{K}$ ). Yamaguchi and 
Kawamura ${ }^{16}$ choose the tunneling part of the Hamiltonian as the perturbing parameter. Ding and $\mathrm{Ng}^{17}$ study the frequency dependence of the noise by means of the equation-of-motion method (also reliable for $T>T_{K}$ ). Meir and Golub 18 perform an exhaustive study of the influence of bias voltage in the shot noise of a quantum dot in the Kondo regime. Dong and Lei ${ }^{19}$ discuss the effect on the shot noise of both external magnetic fields and particle-hole symmetry breaking. Avishai et al ${ }^{20}$ calculate the Fano factor when the leads are $s$-wave superconductors whereas the case of $p$-wave superconductivity is treated by Aono et al ${ }^{21}$ The authors ${ }^{22}$ examine the behavior of the Fano factor at zero temperature when the formation of the Kondo resonance competes with the presence of ferromagnetic leads and spin-flip processes. López et $a l l^{23}$ make use of the two-impurity Anderson Hamiltonian to address the shot noise in double quantum dot systems. To the best of our knowledge, a study of the current fluctuations in a multiprobe Kondo impurity is still missing. This is the gap we want to fill here.

In mesoscopic conductors, Büttiker ${ }^{24}$ shows that the sign of the current cross correlations depends on the statistics of the carriers. It is positive (negative) for bosons (fermions) due to statistical bunching (antibunching). This statement is based on a series of assumptions (e.g., zero-impedance external circuits, spin independent transport, normal thermal leads). Positive correlations can be found if these conditions are not met (see Ref. 25 for references on this subject). Here, we just mention a few studies based on structures involving a quantum dot. Bagret and Nazarov ${ }^{26}$ consider a Coulomb-blockaded quantum dot attached to paramagnetic leads whereas the ferromagnetic case and the spinblockade case are treated by Cottet et al.27 Börlin et $a l^{28}$ and Samuelsson and Büttiker ${ }^{29}$ examine the cross correlations of a chaotic dot in the presence of a superconducting lead. In the spin dependent case, Sánchez et $a l^{30}$ find that the sign of the cross correlations is affected by Andreev cross reflections. In the context of quantum computation, measuring current cross correlations have been shown ${ }^{31}$ to yield a indirect identification of the existence of streams of entangled particles. Therefore, the cross correlations are a valuable tool in characterizing the electron transport in phase-coherent conductors.

In this work, we consider electron transport through a strongly interacting quantum dot attached to three leads. Section [II explains the theoretical framework (slave-boson mean-field theory) we use to compute the conductance and the current-current correlations. We show that the expressions for the cross correlations may be inferred from scattering theory applied to a BreitWigner resonance with renormalized parameters. Section III is devoted to the results. First, we assume that the third lead is a fictitious voltage probe and investigate the effect of dephasing with increasing coupling to the probe. Then, we consider that lead as a real probe and relate the differential conductance measured at one electrode with the LDOS of the artificial Kondo impu- rity. We show next that the sign of the cross correlations of the current is negative, as expected from the fermionic character of the Kondo correlations at very low temperature. Moreover, we discuss the effect of bias voltage, external magnetic fields, and spin-polarized tunneling in the cross correlations. We finish this section with an investigation of the effect of spin polarized transport in the shot noise. Finally, Sec. IV contains our conclusions.

\section{MODEL}

We model the electric transport through the quantum dot using the Anderson Hamiltonian in the limit of large onsite Coulomb interaction $U \rightarrow \infty$. This way we neglect double occupancy in the dot and the Hamiltonian is written in terms of the slave-boson language: ${ }^{32}$

$$
\begin{aligned}
& \mathcal{H}=\sum_{k \alpha \sigma} \varepsilon_{k \alpha \sigma} c_{k \alpha \sigma}^{\dagger} c_{k \alpha \sigma}+\sum_{\sigma} \varepsilon_{0 \sigma} f_{\sigma}^{\dagger} f_{\sigma} \\
& +\sum_{k \alpha \sigma}\left(V_{k \alpha} c_{k \alpha \sigma}^{\dagger} b^{\dagger} f_{\sigma}+\text { H.c. }\right) \\
& +\lambda\left(b^{\dagger} b+\sum_{\sigma} f_{\sigma}^{\dagger} f_{\sigma}-1\right),
\end{aligned}
$$

where $c_{k \alpha \sigma}^{\dagger}\left(c_{k \alpha \sigma}\right)$ is the creation (annihilation) operator describing an electronic state $k$ with spin $\sigma=\{\uparrow, \downarrow\}$ and energy dispersion $\varepsilon_{k \alpha \sigma}$ in the lead $\alpha=\{1,2,3\}, \varepsilon_{0 \sigma}$ is the (spin-dependent) energy level in the dot and $V_{k \alpha}$ is the coupling matrix element. The original dot secondquantization operators have been replaced in Eq. (10) by a combination of the pseudofermion operator $f_{\sigma}$ and the boson field $b$. Hopping off the dot is described by the process $c_{k \alpha \sigma}^{\dagger} b^{\dagger} f_{\sigma}$ : whenever an electron is annihilated by $f_{\sigma}$, an empty state in the dot is created by $b^{\dagger}$ and then $c_{k \alpha \sigma}^{\dagger}$ generates an electron with spin $\sigma$ in the conduction band of contact $\alpha$. The boson operator $b\left(b^{\dagger}\right)$ may be regarded as a projection operator onto the vacuum (empty) state of the quantum dot. To make sure that a state with double occupancy is never created, a constraint with Lagrange multiplier $\lambda$ is added to the Hamiltonian.

The current operator $\hat{I}_{\alpha}$ that yields the electronic flow from lead $\alpha$ is given by

$$
\hat{I}_{\alpha}=\frac{i e}{\hbar}\left[\hat{N}_{\alpha}, \mathcal{H}\right]
$$

where $\hat{N}_{\alpha}=\sum_{k \sigma} c_{k \alpha \sigma}^{\dagger} c_{k \alpha \sigma}$. The general form of the power spectrum of the current fluctuations reads ${ }^{33}$

$$
\begin{aligned}
S_{\alpha \beta}(\omega) & =2 \int d \tau e^{i \omega \tau}\left\langle\left\{\delta \hat{I}_{\alpha}(\tau), \delta \hat{I}_{\beta}(0)\right\}\right\rangle \\
= & 2 \int d \tau e^{i \omega \tau}\left[\left\langle\left\{\hat{I}_{\alpha}(\tau), \hat{I}_{\beta}(0)\right\}\right\rangle-\left\langle\hat{I}_{\alpha}\right\rangle\left\langle\hat{I}_{\beta}\right\rangle\right]
\end{aligned}
$$

$\delta \hat{I}_{\alpha}=\hat{I}_{\alpha}-I_{\alpha}$ describing the fluctuations of the current away from its average value $I_{\alpha}=\left\langle\hat{I}_{\alpha}\right\rangle$. We are interested in the zero-frequency limit of $S_{\alpha \beta}(\omega)$. Since the 


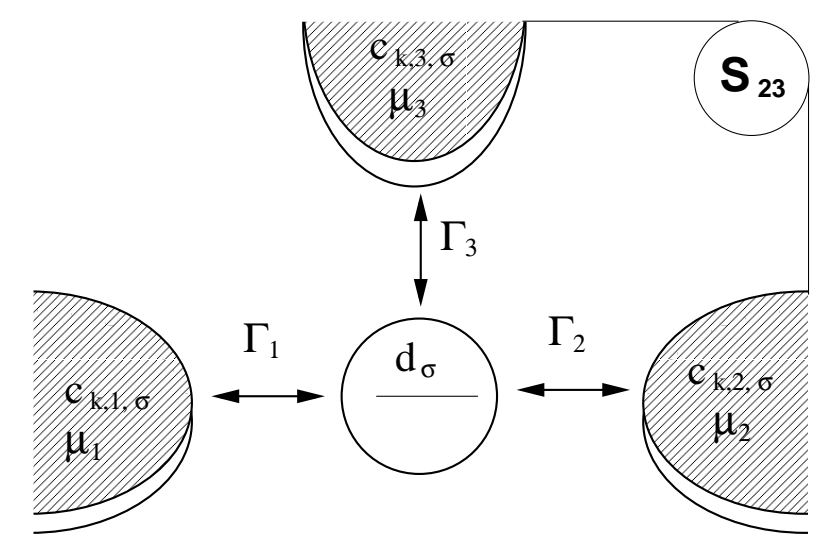

FIG. 1: The system under consideration. The central island is a resonant level coupled to three leads. The level may be shifted through a capacitative coupling to a gate. In the limit of a vanishingly small capacitance, double occupancy in the dot is forbidden and Kondo effect can arise. The currentcurrent cross correlations are measured between leads 2 and 3.

energy scale $k_{B} T_{K}$ in typical experiments is of the order of $100 \mathrm{mK}$, the frequencies should be $\omega \lesssim 2.4 \mathrm{GHz}$. Moreover, we shall work at $T=0$ (see below) so that the current will fluctuate due to quantum fluctuations only (we disregard thermal fluctuations).

\section{A. Mean-field approximation}

The mean-field solution of the Hamiltonian (1) consists of considering the effect of the boson in an averaged way, replacing the true operator $b(t)$ by its expectation value $\langle b(t)\rangle$. Within this approximation the Hamiltonian describes noninteracting quasiparticles with renormalized couplings: $V_{k \alpha} \sqrt{|b|} \rightarrow V_{k \alpha}$. The theory is then suitable for studying the Fermi-liquid fixed point of the Kondo problem (i.e., at $T \ll T_{K}$ ) in which the averaged occupation in the dot is always 1 . The dominant fluctuations in the system are those associated to spin.

The stationary state of the boson field is determined from the $t \rightarrow \infty$ limit of its equation of motion using the Keldysh technique for systems out of equilibrium: ${ }^{34.35}$

$$
\sum_{k \alpha \sigma} \tilde{V}_{k \alpha} G_{f_{\sigma}, k \alpha \sigma}^{<}(t, t)=-i \lambda|b|^{2},
$$

where $G_{f_{\sigma}, k \alpha \sigma}^{<}(t, t)=i\left\langle c_{k \alpha \sigma}^{\dagger}(t) f_{\sigma}(t)\right\rangle$ is the lead-dot lesser Green function. Next, we take into account the constraint:

$$
\sum_{\sigma} G_{f_{\sigma}, f_{\sigma}}^{<}(t, t)=i\left(1-|b|^{2}\right),
$$

$G_{f_{\sigma}, f_{\sigma}}^{<}(t, t)=i\left\langle f_{\sigma}^{\dagger}(t) f_{\sigma}(t)\right\rangle$ being the dot lesser Green function. It yields the nonequilibrium distribution function in the dot.
In evaluating the above Green functions we need the coupling strength given by $\Gamma_{\alpha \sigma}(\epsilon)=\pi \sum_{k}\left|V_{k \alpha}\right|^{2} \delta(\epsilon-$ $\left.\varepsilon_{k \alpha \sigma}\right)$. In the wide band limit, one neglects the energy dependence of $\Gamma$ and the hybridization width is taken as $\Gamma_{\alpha \sigma}=\Gamma_{\alpha \sigma}\left(E_{F}\right)$ for $-D \leq \varepsilon \leq D$ ( $D$ is the high-energy cutoff). We notice that in the presence of Kondo correlations the lifetime broadening becomes renormalized $\Gamma_{\alpha \sigma} \rightarrow \tilde{\Gamma}_{\alpha \sigma}=\pi \sum_{k}\left|\tilde{V}_{k \alpha}\right|^{2} \delta\left(\epsilon-\varepsilon_{k \alpha \sigma}\right)$ and the bare level $\varepsilon_{0 \sigma}$ is shifted to $\tilde{\varepsilon}_{0 \sigma}=\varepsilon_{0 \sigma}+\lambda$. We can now give the full expression of the Fourier-transformed lesser Green function:

$$
G_{f_{\sigma}, f_{\sigma}}^{<}(\epsilon)=2 i \frac{\sum_{\alpha} \tilde{\Gamma}_{\alpha \sigma} f_{\alpha}(\epsilon)}{\left(\epsilon-\tilde{\varepsilon}_{0 \sigma}\right)^{2}+\tilde{\Gamma}_{\sigma}^{2}},
$$

where $\tilde{\Gamma}_{\sigma}=\sum_{\alpha} \tilde{\Gamma}_{\alpha \sigma}$ is the total hybridization width per spin and $f_{\alpha}(\epsilon)=\theta\left(\mu_{\alpha}-\epsilon\right)$ is the Fermi function at zero temperature of lead $\alpha$ with electrochemical potential $\mu_{\alpha}=E_{F}+e V_{\alpha}$. On the other hand, $G_{f_{\sigma}, k \alpha \sigma}^{<}(\omega)$ can be cast in terms of $G_{f_{\sigma}, f_{\sigma}}^{<}(\omega)$ with the help of the equation of motion of the operators and then applying the analytical continuation rules in a complex time contour 34 Therefore, we obtain a closed system of two nonlinear equations [Eqs. (4) and (5)] with unknowns $|b|^{2}$ and $\lambda$ to be found self-consistently.

From the precedent arguments and Eq. (2) we can easily establish an expression for the expectation value of the electric current:

$$
I_{\alpha}=\frac{e}{h} \sum_{\beta \sigma} \int d \epsilon \tilde{T}_{\alpha \beta}^{\sigma}(\epsilon)\left[f_{\alpha}(\epsilon)-f_{\beta}(\epsilon)\right],
$$

which has exactly the same transparent form as the Landauer-Büttiker formula ${ }^{36}$ in the two channel (one per spin) case applied to a double-barrier resonant-tunneling system:

$$
\tilde{T}_{\alpha \beta}^{\sigma}(\epsilon)=\frac{4 \tilde{\Gamma}_{\alpha \sigma} \tilde{\Gamma}_{\beta \sigma}}{\left(\epsilon-\tilde{\varepsilon}_{0 \sigma}\right)^{2}+\tilde{\Gamma}_{\sigma}^{2}},
$$

which has a simple Breit-Wigner lineshape. For the same reason, the quasiparticle density of states is a Lorentzian function centered around the Fermi level (the AbrikosovSuhl resonance). This result is expected since we are dealing with a Fermi liquid but we stress that the physics it contains should not be confused with a noninteracting quantum dot since:

(i) $\tilde{T}$ depends implicitly on $|b|^{2}$ and $\lambda$, and it must then be self-consistently calculated for each set of parameters: contact voltages $\left\{V_{\gamma}\right\}$, magnetic field $\Delta_{Z}=\varepsilon_{0 \uparrow}-\varepsilon_{0 \downarrow}$, gate voltage $\varepsilon_{0}\left(V_{g}\right)$, and lead magnetization.

(ii) $\tilde{T}$ is renormalized by Kondo correlations (as the bare $\Gamma$ and $\varepsilon_{0}$ are),

(iii) $\tilde{T}$ has a nontrivial dependence on the bias voltage.

All these features give rise to a number of effects that are not seen in a noninteracting resonant-tunneling diode. There are many instances: regions of negative differential conductance in the current-voltage characteristics of a double quantum dot, $\stackrel{37}{a}$ a crossover from 
Kondo physics to an antiferromagnetic singlet in the twoimpurity problem ${ }^{23}$ an anomalous sign of the zero-bias magnetoresistance, ${ }^{22}$ etc. Below, we shall discuss another example without counterpart in a noninteracting BreitWigner resonance: When the Kondo peak splits due to a large bias voltage.

\section{B. Current-current correlations}

We consider now the current fluctuations given by Eq. (3) at zero frequency $S_{\alpha \beta}(0)$. To simplify the notation we introduce $G_{0}(\omega)=G_{f_{\sigma}, f_{\sigma}}(\omega)$ as the dot Green function. After lengthy algebra, we have

$$
\begin{gathered}
S_{\alpha \beta}(0)=\frac{4 e^{2}}{h} \int d \epsilon \tilde{\Gamma}_{\alpha} \tilde{\Gamma}_{\beta}\left[G_{0}^{<} G_{0}^{>}-G_{0}^{a} G_{0}^{>} f_{\alpha}\right. \\
+G_{0}^{<} G_{0}^{a}\left(1-f_{\beta}\right)-G_{0}^{<} G_{0}^{r}\left(1-f_{\alpha}\right)+G_{0}^{r} G_{0}^{>} f_{\beta}-G_{0}^{a} G_{0}^{a} f_{\alpha}\left(1-f_{\beta}\right) \\
\left.-G_{0}^{r} G_{0}^{r} f_{\beta}\left(1-f_{\alpha}\right)-i \frac{\delta_{\alpha \beta}}{\pi \tilde{\Gamma}_{\alpha}}\left(G_{0}^{<}\left(1-f_{\beta}\right)-G_{0}^{>} f_{\alpha}\right)\right] .
\end{gathered}
$$

This formula (or variations of it) has been already employed in the literature. Wei et al ${ }^{38}$ prove it using the Fisher-Lee-Baranger-Stone relation ${ }^{39}$ to write the scattering matrix elements in terms of the retarded Green function of the dot, $G_{0}^{r}$. Dong and Lei19 ${ }^{19}$ and López et $a l^{22.23}$ find it in Kondo problems within a slave-boson mean-field framework. Actually, in Ref. 23 it is shown that the shot noise in a two-terminal geometry reads $S \sim \tilde{T}(1-\tilde{T})$, i.e., the well known result for the partition noise but with renormalized transmissions. Souza et al $\stackrel{40}{=}$ calculate the noise of an ultrasmall magnetic tunnel junction by means of Eq. (9) within a Hartree-Fock framework. In general, we can say that Eq. (9) is consistent within mean-field theories. However, some caution is needed if one wishes to go beyond a mean-field level. In deriving Eq. (9), one needs to apply Wick theorem, which is valid only for noninteracting (quasi)-particles. More specifically, one finds terms that read:

$$
\begin{aligned}
& \left\langle c_{k \alpha \sigma}^{\dagger}(t) f_{\sigma}(t) c_{k \beta \sigma^{\prime}}^{\dagger}(0) f_{\sigma^{\prime}}(0)\right\rangle= \\
& \left\langle c_{k \alpha \sigma}^{\dagger}(t) f_{\sigma}(t)\right\rangle\left\langle c_{k \beta \sigma^{\prime}}^{\dagger}(0) f_{\sigma^{\prime}}(0)\right\rangle \\
& \quad+\left\langle c_{k \alpha \sigma}^{\dagger}(t) f_{\sigma^{\prime}}(0)\right\rangle\left\langle f_{\sigma}(t) c_{k \beta \sigma^{\prime}}^{\dagger}(0)\right\rangle .
\end{aligned}
$$

The first term in the left-hand side corresponds to disconnected diagrams that cancel out the term $\left\langle\hat{I}_{\alpha}\right\rangle\left\langle\hat{I}_{\beta}\right\rangle$ of Eq. (3) whereas the second term contributes to Eq. (9). Therefore, the particular Hamiltonian has to be cast first in a quadratic form. Zhu and Balatsky ${ }^{41}$ incorrectly state that Eq. (9) takes into account the many-body effects. Also, it is not clear how this formula is inferred within the equation-of-motion method employed by Lü and Liu ${ }^{42}$

In our case, the mean-field approximation is known to be the leading term in a $1 / N$ expansion, ${ }^{43}$ where $N=2$ is the spin degeneracy. Therefore, we neglect the fluctuations of both the boson field $(\delta b=0)$ and the renormalization of the resonant level $(\delta \lambda=0), \frac{19.32}{,}$ which could be calculated in the next order. This is valid as long as we restrict ourselves to the Fermi-liquid fixed point of the Kondo problem. We are not aware of real $1 / N$ correction calculations of shot noise. Although Meir and Golub 18 perform a noncrossing approximation (NCA), they just substitute the NCA propagators into Eq. (9), with the limitations exposed above.

The current-current correlations can be deduced either using Eq. (9) or using the scattering approach for the multiterminal case (see Ref. 24). The latter formalism amounts to replacing the bare parameters by the renormalized ones ${ }^{23}$. We consider the illustrative case of having different electrochemical potentials in two leads, $\mu_{\alpha} \neq \mu_{\beta}$ (e.g., $\alpha=2$ and $\beta=3$ ) at zero temperature. We find

$$
S_{23}(0)=-\frac{2 e^{2}}{h} \sum_{\gamma, \delta} \int d \epsilon \operatorname{Tr}\left(s_{2 \gamma}^{\dagger} s_{2 \delta} s_{3 \delta}^{\dagger} s_{3 \gamma}\right)\left(f_{\gamma}-f_{a}\right)\left(f_{\delta}-f_{b}\right),
$$

where $s_{\alpha \beta}$ is the renormalized scattering amplitude of a Breit-Wigner resonance:

$$
s_{\alpha \beta}^{\sigma}(\epsilon)=\delta_{\alpha \beta}-\frac{2 i \sqrt{\tilde{\Gamma}_{\alpha \sigma} \tilde{\Gamma}_{\beta \sigma}}}{\epsilon-\tilde{\varepsilon}_{0 \sigma}+i \tilde{\Gamma}_{\sigma}} .
$$

In Eq. (11) the trace $\operatorname{Tr}(\ldots)$ is over spin indices. The Fermi functions $f_{a}$ and $f_{b}$ are arbitrary $\stackrel{24}{\underline{\underline{24}}}$ Choosing $f_{a}=$ $f_{b}=f_{3}$, we obtain

$$
\begin{aligned}
& S_{23}(0)=-\frac{2 e^{2}}{h} \sum_{\sigma} \int d \epsilon\left\{\tilde{T}_{12}^{\sigma} \tilde{T}_{13}^{\sigma}\left[f_{1}-f_{3}\right]^{2}\right. \\
& \left.+\tilde{R}_{22}^{\sigma} \tilde{T}_{32}^{\sigma}\left[f_{2}-f_{3}\right]^{2}+2 \tilde{T}_{12}^{\sigma} \tilde{T}_{23}^{\sigma}\left[f_{1}-f_{3}\right]\left[f_{2}-f_{3}\right]\right\},
\end{aligned}
$$

where $R_{22}^{\sigma}$ is the reflection probability (in general $R_{\alpha \alpha}=$ $\left.1-\sum_{\beta} T_{\alpha \beta}\right)$. Notice that generally one cannot write the multilead current-current correlations only in terms of transmission probabilities as in Eq. (13). This was firstly pointed out by Büttiker, ${ }^{44}$ suggesting the appearance of exchange effects in noise measurements. Here, since we are dealing with a (renormalized) Breit-Wigner resonance, exchange corrections due to phase differences do not play any role.

For completeness, we give now the formula for the shot noise, i.e., the current-current correlations measured at the same lead (e.g., lead 1). Following the way of reasoning that led to Eq. (13) we obtain

$$
\begin{aligned}
S_{11}(0)= & \frac{2 e^{2}}{h} \sum_{\sigma} \int d \epsilon\left\{\tilde{T}_{12}^{\sigma} \tilde{R}_{11}^{\sigma}\left[f_{1}-f_{2}\right]^{2}\right. \\
& \left.+\tilde{T}_{13}^{\sigma} \tilde{R}_{11}^{\sigma}\left[f_{1}-f_{3}\right]^{2}+\tilde{T}_{12}^{\sigma} \tilde{T}_{13}^{\sigma}\left[f_{2}-f_{3}\right]^{2}\right\} .
\end{aligned}
$$

\section{RESULTS}

In the following, we present results obtained by selfconsistently solving Eqs. (4) and (5) for each bias volt- 
age. The rest of parameters is changed in the next subsections. Throughout this work, we have checked that current conservation $\left(I_{1}+I_{2}+I_{3}=0\right)$ is fulfilled. ${ }^{45}$

Tunneling effects are incorporated at all orders since at equilibrium the Kondo temperature is found to be

$$
k_{B} T_{K}^{0}=\tilde{\Gamma}=D \exp \left(-\pi\left|\varepsilon_{0}\right| / 2 \Gamma\right),
$$

which is clearly a nonperturbative result. In Eq. 15 $\Gamma=\sum_{\alpha=1}^{3} \Gamma_{\alpha}$ is the total hybridization broadening. The reference energy will be always set at $E_{F}=0$ and the energy cutoff is $D=100 \Gamma$. The bare level is $\varepsilon_{0}=-6 \Gamma$, deep below $E_{F}$ to ensure a pure Kondo regime.

\section{A. Dephasing}

Before turning to the determination of current cross correlators, we briefly discuss with an application the capabilities of three-terminal setups to illustrate some difficult aspects of the physics of the two-terminal Kondo effect. As mentioned in the Introduction, we investigate the action of a fictitious voltage probe 46 (say, lead 3) in order to simulate decoherence effects on the formation of the Kondo resonance between leads 1 and 2.47 These contacts play the role of source and drain, respectively. The voltage probe mode ${ }^{46}$ describes decoherence since an electron that is absorbed into the probe looses its coherence. The exiting electron is replaced by an electron (with an unrelated phase) injected by the probe.

At low temperatures the principal source of dephasing is due to quasi-elastic scattering. ${ }^{48}$ We consider then a voltage probe that preserves energy ${ }^{49}$ The current through the voltage probe is zero at every energy $\epsilon$. Thus, from Eq. (7) the distribution function at the probe reads

$$
f_{3}(\epsilon)=\frac{T_{13}(\epsilon) f_{1}(\epsilon)+T_{23}(\epsilon) f_{2}(\epsilon)}{T_{13}(\epsilon)+T_{23}(\epsilon)} .
$$

We have to insert this result into Eqs. (4) and (5) and solve self-consistently for the hybridization couplings $\tilde{\Gamma}$ and the resonance level $\tilde{\varepsilon}_{0}$ in the presence of quasi-elastic scattering for each value of the applied bias voltage. Then we compute numerically the differential conductance $G=$ $d I / d V_{\text {sd }}$, where $I=I_{1}=-I_{2}$ and $V_{\text {sd }}=V_{1}-V_{2}$.

Figure 2(a) shows $G$ for different values of the coupling to the probe (we set $\Gamma_{2}=\Gamma_{1}$ ). For $\Gamma_{3}=0$ we obtain the well known zero-bias anomaly, which arises from the formation of the Kondo resonance at $V_{\text {sd }}=0$. As $\Gamma_{3}$ increases we observe a quenching of the Kondo peak. The degree of the conductance suppression depends on the coupling to the probe. At each bias, $\mu_{3}$ (which has to be self-consistently calculated) adjusts itself to fulfill the condition of zero net current at each energy $\epsilon$. Hence, $\Gamma_{3}$ is a phenomenological parameter that includes dephasing processes present in the quantum dot. To see this, we can write down the current through, say, lead 1, using

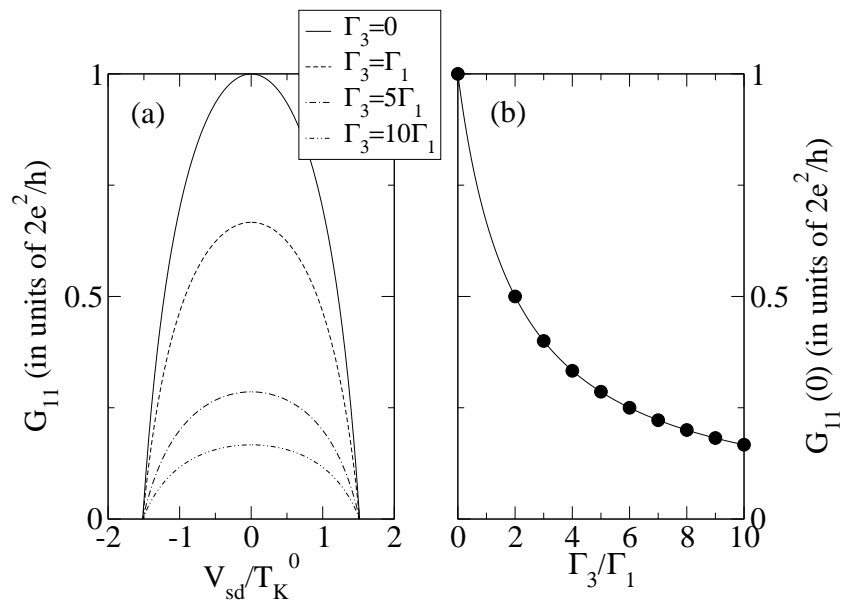

FIG. 2: (a) Differential conductance $G_{11}$ versus bias voltage $V_{1}$ as a function of the bare coupling $\Gamma_{3}$ to the voltage probe (reservoir 3) for $\Gamma_{1}=\Gamma_{2}$ and $\varepsilon_{0}=-6 \Gamma$. (b) Linear conductance $G_{11}(0)$ showing the reduction of the peak in (a) versus the coupling to the voltage probe. The dots are numerical results where the line corresponds to an analytical formula (see text).

Eqs. (7) and (16):

$$
I=\frac{e}{\hbar} \frac{4 \tilde{\Gamma}_{1} \tilde{\Gamma}_{2}}{\tilde{\Gamma}_{1}+\tilde{\Gamma}_{2}} \int d \epsilon A_{0}(\epsilon)\left[f_{1}(\epsilon)-f_{2}(\epsilon)\right],
$$

where $A_{0}(\varepsilon)=-\operatorname{Im} G_{0}^{r}(\varepsilon) / \pi$ is the LDOS in the dot. Equation (17) has the form of a formula for a twoterminal current ${ }^{50}$ with $G_{0}^{r}(\varepsilon)=\left[\varepsilon-\tilde{\varepsilon}_{0}+i\left(\tilde{\Gamma}_{1}+\tilde{\Gamma}_{2}+\right.\right.$ $\left.\left.\tilde{\Gamma}_{3}\right)\right]^{-1}$. It is straightforward to show that a nonzero $\Gamma_{3}$ leads to deviations of Eq. (17) from the unitary limit.

In Fig. 2(b) we plot the linear conductance $\mathcal{G}=$ $G\left(V_{\mathrm{sd}}=0\right)$ as a function of $\Gamma_{3} / \Gamma_{1}$ from the results found numerically. At zero bias we can find from Eq. (17) an analytical expression for the reduction of the peak:

$$
\mathcal{G}=\frac{2 e^{2}}{h} \frac{2}{2+\Gamma_{3} / \Gamma_{1}} .
$$

It is shown in Fig. 2(b) (full line). In the limit of $\Gamma_{3} / \Gamma_{1} \ll 1$ a similar expression for the reduction of the peak was found by Kaminski et al.,,$\frac{4}{4}$ the source of decoherence being an ac voltage applied to the dot level.

\section{B. Multiterminal conductance}

From now on, we consider lead 3 as a real electrode with tunable voltage $V_{3}$. We set $V_{3}=V_{2}=0$ and vary the tunneling coupling $\Gamma_{3}$. The self-consistent results of Eqs. (4) and (5) are inserted in Eqs. (7) to calculate the differential conductance through lead 1: $G_{11}=d I_{1} / d V_{1}$. Figure[3(a) shows $G_{11}$ as a function of $V_{1}$. At $\Gamma_{3}=0$ the conductance at $V_{1}=0$ achieves the unitary limit as in the two-terminal case. With increasing the coupling to 


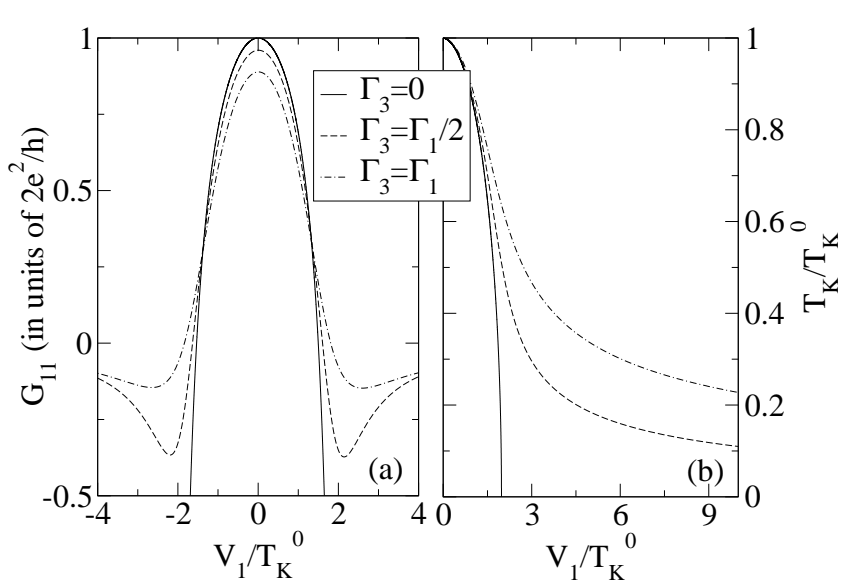

FIG. 3: (a) Differential conductance $G_{11}$ versus bias voltage $V_{1}$ for $\varepsilon_{0}=-6 \Gamma\left(T_{K}^{0}=8 \times 10^{-3} \Gamma\right)$. (b) Dependence of the Kondo temperature on $V_{1}$.

third lead, $G_{11}(0)$ decreases. For equal tunnel couplings $\left(\Gamma_{1}=\Gamma_{2}=\Gamma_{3}=\Gamma / 3\right), G_{11}(0)$ does not reach 1 (in units of $\left.2 e^{2} / h\right)$ but instead $G_{11}(0)=8 / 9$, in agreement with Ref. 51. This is an immediate consequence of having three leads with identical couplings. Interestingly, the Kondo temperature of Fig. 3 (b) does not vanish abruptly for $V_{1}=2 T_{K}^{0}$, as known in the two-terminal case (see the case $\Gamma_{3}=0$ ). This is an important result as it implies that Kondo correlations survive at large voltages. The effect is reminiscent of the situation found by Aguado and Langreth ${ }^{37}$ in tunnel-coupled double quantum dots, though the physical origin is clearly distinct.

\section{Sign of the current cross-correlations.}

Comparison with a noninteracting quantum dot

We now focus on the current-current correlations of the current for $V_{3}=V_{2}=0$ and equal couplings $\Gamma_{1}=$ $\Gamma_{2}=\Gamma_{3}=\Gamma / 3$. Later, we shall allow for nonzero voltage differences between leads 2 and 3. In Fig. 世(a), we show the cross correlator $S_{23}(0)$ obtained from Eq. (13). As expected, $S_{23}$ is zero for $V_{1}=0$ and negative elsewhere. This reflects the fermionic nature of the quasiparticles. For comparison, we plot in Fig. प(b) the corresponding $S_{23}$ for a noninteracting resonant double-barrier structure with the level at $E_{F}$ (of course, for $\varepsilon_{0}=-6 \Gamma$ the spectrum $S_{23}$ is always very small as the transmission is). In this case, the physics is governed by the bare coupling $\Gamma, 52$ On the contrary, in the Kondo problem the dominating energy scale is $T_{K}$. Qualitatively, Fig. U(a) and 4 (b) look the same until $V_{1} \sim 2 T_{K}$. The cross correlator in the Kondo case increases with voltage while in the noninteracting case $S_{23}$ saturates at large voltages. It is easy to show that the saturation value is given by $-8 \pi / 81 \simeq-0.31$ (in units of $4 e^{2} \Gamma / h$ ). The reason for the increase of $S_{23}(0)$ in Fig. 目(a) is that $T_{K}$ is voltage dependent unlike the bare $\Gamma$, even in the wide-band limit.

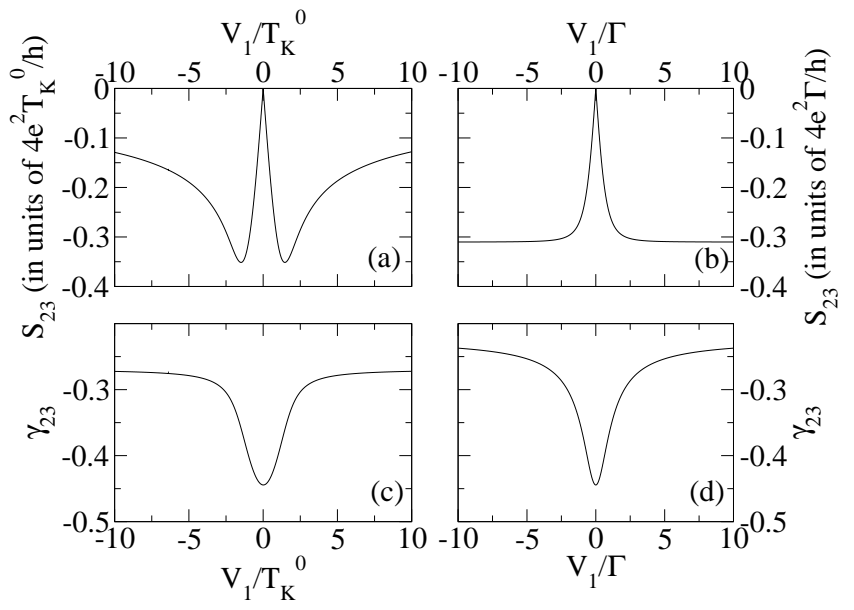

FIG. 4: (a) Current-current cross correlation measured in leads 2 and $3, S_{23}(0)$, as a function of the bias voltage in the injecting lead, $V_{1}$. Kondo correlations involve an increase of $S_{23}(0)$ for voltages larger than $2 T_{K}$. (b) Same as (a) for a noninteracting quantum dot with a resonant level exactly at $E_{F} . \quad(\mathrm{c})$ and $(\mathrm{d})$ correspond to the Fano factor $\gamma_{23}$ as a function of voltage for the interacting and noninteracting case respectively.

In particular, the current-voltage characteristics shows a region of negative differential conductance in the Kondo case [see Fig. 31(a)] whereas it reaches a constant value at large voltages for an noninteracting quantum dot.

To avoid effects due to moderate biases, in what follows we shall concentrate on a normalized $S_{23}$. We define the Fano factor of $S_{23}$ as

$$
\gamma_{23}=\frac{S_{23}}{2 e \sqrt{\left|I_{2}\right|\left|I_{3}\right|}} .
$$

If the scattering region were a simple barrier of transmission $T, \gamma_{23}$ would be simply -1 . This number changes when the system under consideration is a quantum dot. In Figs. 4 (a) and (b), we plot $S_{23}$ for the Kondo and the noninteracting case, respectively. Their corresponding Fano factors are shown in Figs. I( (c) and (d). We see that $\gamma_{23}$ has a minimum at $V_{1}=0$. Analytically, we find $\gamma_{23}(0)=-4 / 9 \simeq-0.44$, which is in excellent agreement with the numerical result. Likewise, we can assess the limit of $\gamma_{23}$ at very high voltages $\left(V_{1} \gg T_{K}^{0}\right)$. We get $\gamma_{23}=-2 / 9 \simeq-0.22$. As observed, both curves tend to this value, though for a noninteracting quantum dot it is more quickly due to the independence of $\Gamma$ on the bias voltage.

\section{Effect of the nonequilibrium splitting on the current-current correlations}

Now we turn to an exciting case. Consider the following bias configuration: $V_{2}=-V_{3} \neq 0$ and determine the differential conductance $G_{11}$ as a function of $V_{1}$. The case $V_{2}=-V_{3}=0$ has been treated before. However, due to 


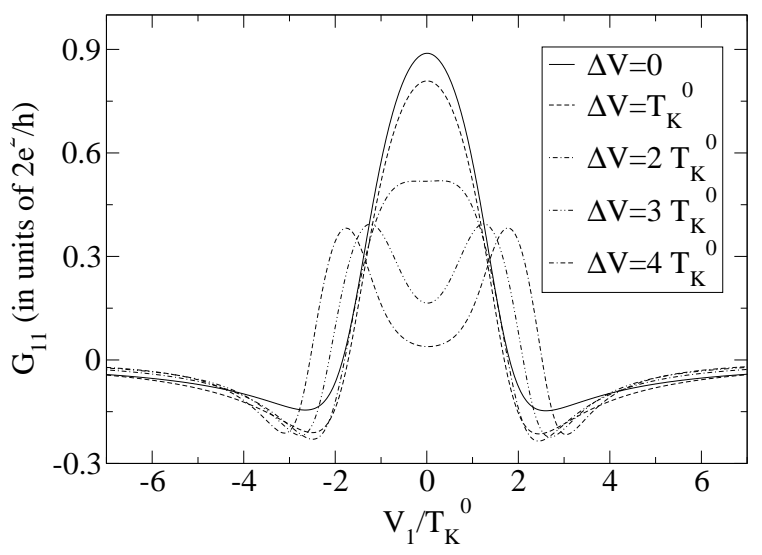

FIG. 5: Differential conductance $G_{11}$ versus bias voltage $V_{1}$ for different values of the voltage difference $\Delta V \equiv\left|V_{2}-V_{3}\right| \gtrsim$ $2 T_{K}^{0}$.

the fact that the boson field never vanishes, we can study the situation $\Delta V \equiv\left|V_{2}-V_{3}\right| \gtrsim 2 T_{K}^{0}$. As remarked in the Introduction, it has been argued 11.12 and experimentally observed $^{13}$ that in a three-lead geometry the splitting of the Kondo resonance due to voltage is visible, unlike the two-terminal case. Moreover, in Refs. 12 51] it has been noticed that the conductance $G_{11}$ is not sensitive to the strength of the coupling to the third lead, showing always a two-peak structure. Of course, only when the third lead is weakly coupled to the $\operatorname{dot} G_{11}$ is a measure of the LDOS. But since we are interested in the transport properties of the system, our choice of equal coupling constants does not affect the results for the conductances and the current-current correlations.

In Fig. 5] we plot the behavior of the differential conductance $G_{11}$. At $\Delta V=0$ we obtain the zero-bias anomaly of Fig. 3(a). As $\Delta V$ increases, $G_{11}$ is split at $V_{2} \sim T_{K}^{0}$. Both splitting peaks lie at $V_{1} \sim V_{3}$ and $V_{1} \sim V_{2}$, i.e., when a pair of electrochemical potentials are aligned. It is also at those points where the Kondo temperature is larger. We emphasize that this effect has no similitude in the electronic transport through a noninteracting quantum dot. Still, a mean-field theory of the Kondo effect as presented here is able to capture this physics. At the same time that the splitting in $G_{11}$ develops, the height of the peaks decreases, suppressing the zero-bias anomaly, although not so strongly as in the experiment ${ }^{13}$ due to the absence of inelastic scattering in this case.

We now use Eq. (13) to calculate the cross correlations between leads 2 and 3 . The results are presented in Fig. 6(a). The dependence of $S_{23}$ on voltage is rather asymmetric, hindering the observation of a clear indication due to the voltage induced splitting. The asymmetry is caused by the third term of the right-hand side of Eq. (13), which is not symmetric under the operation $V_{1} \rightarrow-V_{1}$ when $\Delta V>0$. That is the reason why we

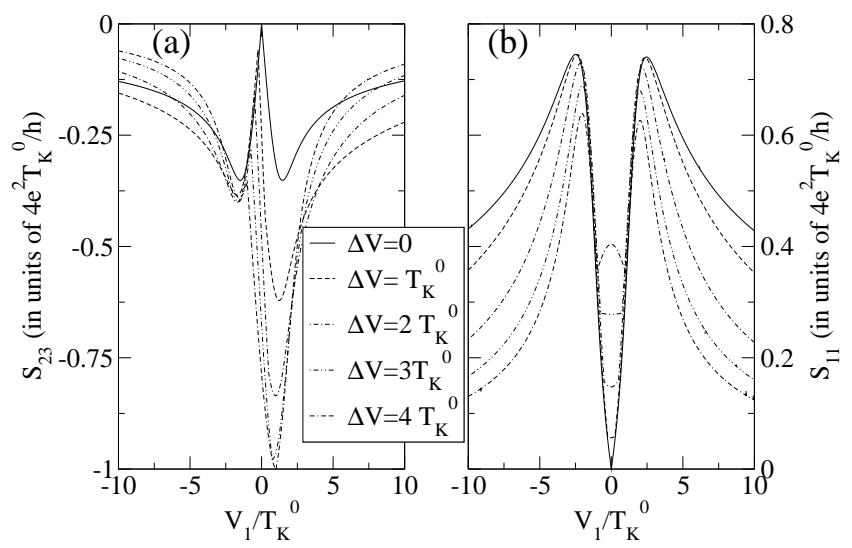

FIG. 6: (a) Cross correlations of the current measured between leads 2 and 3 for the case treated in Fig. 5 (b) Same as (a) for the shot noise in lead 1.

next consider the shot noise in lead $1 S_{11}$, which is an even function of the applied $V_{1}$.

In Fig. 6 (b) we plot the results of Eq. (14). We observe that $S_{11}$ at $V_{1}=0$ is nonvanishing with increasing $\Delta V$, causing a divergence of the Fano factor. This is not related to the Kondo physics but with the fact that the lead 1 at $V_{1}=0$ acts as a voltage probe with zero impedance since the net current flowing through it is zero. Including the fluctuations of the potentials would probably cancel out the divergence. A consequence of Kondo physics is that the minimum at $V_{1}=0$ turns into a maximum. This occurs when the splitting in $G_{11}$ is sharply formed [see Fig 5 .

\section{E. Spin dependent transport and current cross correlations}

So far we have assumed spin-independent transport. Let us go back to the bias configuration of Secs. IIIB and IIIC $\left(V_{2}=V_{3}=0\right)$ and focus on the spin-dependent transport properties. It is customary in the theoretical studies of spintronic transport to take into account the influence of external magnetic fields and ferromagnetic electrodes, among other parameters $\$ 53$ Firstly, we shall change the external Zeeman field and then enable the presence of spin-polarized tunneling.

\section{Magnetic field}

We assume that the leads are paramagnetic and that the magnetic field is applied only to the dot, resulting in a Zeeman gap of the bare resonant level: $\Delta_{Z}=\varepsilon_{0 \uparrow}-$ $\varepsilon_{0 \downarrow}$. It is well known that, as a consequence, the Kondo resonance is split when $\Delta_{Z} \sim T_{K}^{0} \underline{\underline{7}}$

Figure [1(a) shows the differential conductance $G_{11}$ for different values of the Zeeman field. The conductance is split and quenched with increasing $\Delta_{Z}$, as expected. In 


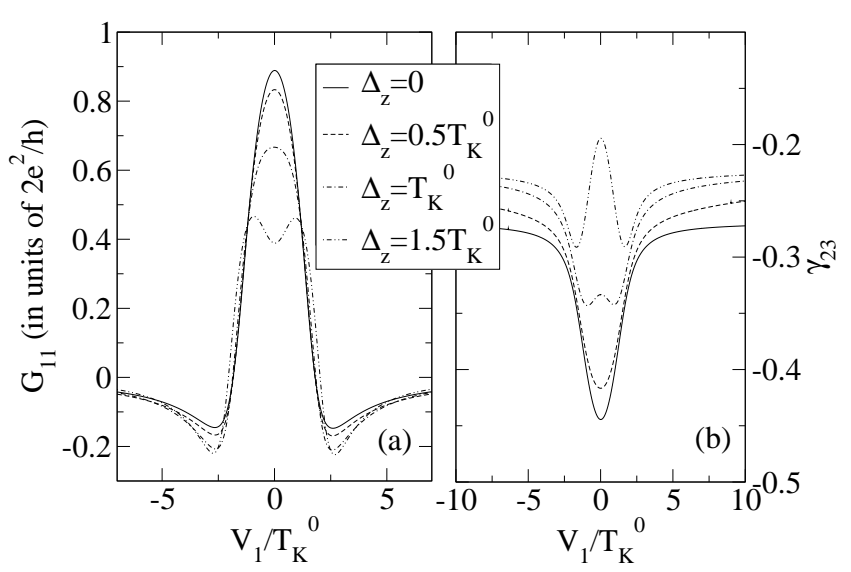

FIG. 7: (a) Differential conductance $G_{11}$ versus $V_{11}$ as a function of the Zeeman term $\Delta_{Z}$ for $V_{2}=V_{3}=0$. (b) Same as (a) for the Fano factor of the cross correlator, $\gamma_{23}$.

Fig. 7 (b), we depict the Fano factor of the cross correlator $\gamma_{23}$. It exhibits a very interesting feature. Due to the splitting of the Kondo peak, the minimum of the cross correlator at $V_{1}=0$ becomes a local maximum, resulting from the suppression of the Kondo effect. However, this change occurs before the splitting of the conductance $G_{11}$. Therefore, measuring the shot noise provides new information in this case. The presence of the splitting would be detected in an experiment more precisely by means of the shot noise. The underlying reason is that the form of Eq. (13) differs from that of the current which is basically proportional to $\tilde{T}_{12}$ alone, see Eq. (7). As a result, the width of the $G_{11}$ resonance is a bit larger than the $\gamma_{23}$ antiresonance and the former is then more robust than the latter against the application of magnetic fields.

\section{Ferromagnetic leads}

There has recently been considerable debate about the influence of ferromagnetic leads in the Kondo physics of a quantum dot 54.55 .56 .57 In the preceding subsection, it was clear that an external magnetic field alters the real part of the quantum-dot self-energy, breaking the spin degeneracy. In the case of spin polarized tunneling, the situation is more subtle ${ }^{57}$ When the magnetic moments of the contacts are aligned along the same direction, the density of states of the localized electron undergoes a splitting if particle-hole symmetry is broken ${ }^{58}$ Recent transport experiments with $\mathrm{C}_{60}$ molecules and carbon nanotubes have addressed this regime 59.60 However, in our case the dot is in the strong coupling limit and the Kondo effect is pure in the sense that no charge fluctuations are allowed. Thus, no splitting is expected in the differential conductance.

In Fig. 8(a), we show the cross correlator $\gamma_{23}$ for different values of the lead magnetization in the parallel

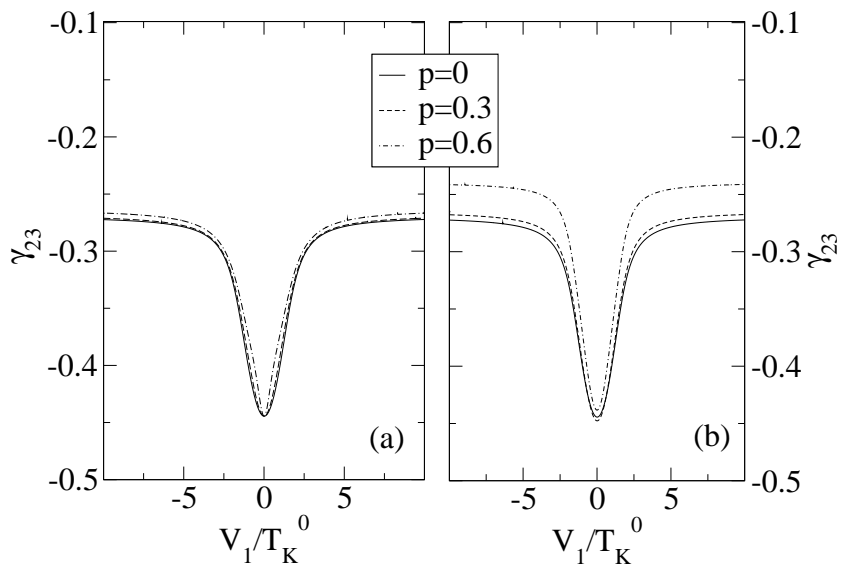

FIG. 8: Fano factor of the cross correlator, $\gamma_{23}$ vs $V_{1} / T_{K}^{0}$ for different lead magnetization when $V_{2}=V_{3}=0$. (a) Parallel alignment between the magnetizations of the leads with spin polarizations: $p_{1}=p_{2}=p_{3}=p$. (b) Antiparallel case with $p_{1}=-p_{2}=-p_{3}=p$.

case. This means that $p_{1}=p_{2}=p_{3}=p$, where $p_{\alpha}$ is the spin polarization of lead $\alpha$. Ferromagnetism in the leads arises through spin-dependent densities of states $\nu_{\alpha \sigma}(\epsilon)=\sum_{k} \delta\left(\epsilon-\varepsilon_{k \alpha \sigma}\right)$. Hence, the linewidths become spin dependent: $\Gamma_{\alpha \sigma}=\left(1 \pm p_{\alpha}\right) \Gamma_{\alpha}$, where $+(-)$ corresponds to up (down) spins. We prefer to restrict $p_{\alpha}$ to small values as strong magnetizations would require a proper treatment of the reduction of the bandwidth $D$. We observe that $\gamma_{23}$ is rather insensitive to changes in $p$ in the same fashion as $G_{11}$ is in the Fermi-liquid fixed point $^{22}$. Only at moderate polarizations $(p=0.6)$ we see that the dip in $\gamma_{23}$ gets narrower because the Kondo temperature decreases as $p$ increases 56.57 . In addition, $\gamma_{23}$ is always negative in contrast to the results obtained in the Coulomb blockade regime where $\gamma_{23}$ can take positive values 27 . When the spin-flip scattering rate is smaller than the tunneling rate, $\gamma_{23}$ can be positive. However in the Kondo regime this condition is never met since the rate of spin flip scattering $\sim 1 / T_{K}$ is always much longer than the tunneling rate $\sim 1 / \Gamma$. Figure 8 (b) is devoted to the antiparallel case: $p_{1}=-p_{2}=-p_{3}=p$. Accordingly, $\gamma_{23}$ is lifted with increasing lead polarization since the conductance peak decreases with increasing $p$ (roughly, with a factor $\left.1-p^{2}\right)^{22}$.

\section{CONCLUSION}

In summary, we have investigated the Kondo temperature, the differential conductance and cross correlations of the current when three leads are coupled to an artificial Kondo impurity in the Fermi-liquid fixed point of the infinite- $U$ Anderson Hamiltonian $\left(T \ll T_{K}\right)$. We have performed a systematic study of the properties of the cross correlators when dc bias, Zeeman splittings, and ferromagnetic leads influence the nonequilibrium transport through the quantum dot. Our most relevant result 
is the behavior of the shot noise when there arises a voltage induced splitting in the quantum dot.

In addition, we have studied the current of a twoterminal quantum dot attached to a voltage probe. We have shown that increasing the coupling with the probe induces a quenching of the Kondo peak. Despite the simplicity of this approach, it gives rise to results that are in agreement with more sophisticated models $\stackrel{4.61}{=}$ though the precise processes responsible for the decoherence need still to be derived from a microscopic model.

We have not exhausted all the possibilities that the model offers and more complicated geometries with appealing results can be envisaged. One could address the situation with two injecting and two receiving leads, which could give rise to Hanbury Brown-Twiss-like effects ${ }^{62}$ We expect that phase related exchange terms will arise especially at higher temperatures $\left(T>T_{K}\right)$, when the singlet state between the localized spin and the conduction electrons is not yet well formed. We believe that in the presence of spin-polarized couplings due to ferromagnetic leads, bunching effects will be enhanced ${ }^{63}$

Improvements of the model should go in the direction of including fluctuations of the boson field and of the renormalized level. However, we do not expect large deviations from the results reported here when $T \ll T_{K}$. These fluctuations will evidently become important as temperature approaches $T_{K}$. Experimentally, our predictions can be tested with present technology such as GaAs quantum dots 13 or carbon-nanotube nanostructures ${ }^{64}$

\section{Acknowledgements}

We gratefully acknowledge R. Aguado, M. Büttiker, S. Pilgram and P. Samuelsson for helpful comments. This work was supported by the EU RTN under Contract No. HPRN-CT-2000-00144, Nanoscale Dynamics, and by the Spanish MECD.
1 A.C. Hewson, The Kondo Problem to Heavy Fermions (Cambridge University Press, Cambridge, UK, 1993).

2 T.K. Ng and P.A. Lee, Phys. Rev. Lett. 61, 1768 (1988); L.I. Glazman and M.E. Raikh, JETP Lett. 47, 452 (1988).

3 D. Goldhaber-Gordon, H. Shtrikman, D. Mahalu, D. Abusch-Magder, U. Meirav, and M.A. Kastner, Nature (London) 391, 156 (1998); S.M. Cronenwett, T. H. Oosterkamp, and L. P. Kouwenhoven, Science 281, 540 (1998); J. Schmid, J. Weis, K. Eberl, and K. v. Klitzing, Physica B 256-258, 182 (1998).

4 A. Kaminski, Yu.V. Nazarov, and L.I. Glazman, Phys. Rev. Lett. 83, 384 (1999); ibid. Phys. Rev. B 62, 8154 (2000).

5 P. Coleman, C. Hooley, and O. Parcollet, Phys. Rev. Lett. 86, 4088 (2001).

6 A. Rosch, J. Paaske, J. Kroha, and P. Wölfle, Phys. Rev. Lett. 90, 07684 (2003);

7 Y. Meir, N.S. Wingreen and P.A. Lee, Phys. Rev. Lett. 70, 2601 (1993); N.S. Wingreen and Y. Meir, Phys. Rev. B 49, 11040 (1994).

8 J. König, J. Schmid, H. Schoeller, and G. Schön, Phys. Rev. B 54, 16820 (1996).

9 A. Rosch, J. Kroha, and P. Wölfle, Phys. Rev. Lett. 87, 156802 (2001);

10 T. Fujii and K. Ueda, Phys. Rev. B 68, 155310 (2003).

11 Q.-f. Sun and H. Guo, Phys. Rev. B 64, 153306 (2001).

12 E. Lebanon and A. Schiller, Phys. Rev. B 65, 035308 (2001).

13 S. De Franceschi, R. Hanson, W.G. van der Wiel, J.M. Elzerman, J.J. Wijpkema, T. Fujisawa, S. Tarucha, and L.P. Kouwenhoven, Phys. Rev. Lett. 89, 156801 (2002).

14 For a complete review, see Ya. M. Blanter and M. Büttiker, Phys. Rep. 336, 1 (2000).

15 S. Hershfield, Phys. Rev. B 46, 7061 (1992).

16 F. Yamaguchi and K. Kawamura, J. Phys. Soc. Jap. 63 1258 (1994).

17 G.-H. Ding and T.-K. Ng, Phys. Rev. B 56, R15 521 (1997).
18 Y. Meir and A. Golub, Phys. Rev. Lett. 88, 116802 (2002).

19 B. Dong and X.L. Lei, J. Phys.: Condens. Matter 14, 4963 (2002).

20 Y. Avishai, A. Golub, and A.D. Zaikin, Phys. Rev. B 67, 041301(R) (2003).

21 T. Aono, A. Golub, and Y. Avishai, Phys. Rev. B 68, 045312 (2003).

22 R. López and D. Sánchez, Phys. Rev. Lett. 90, 116602 (2003).

23 R. López, R. Aguado, and G. Platero, Phys. Rev. B 69, 235305 (2004).

24 M. Büttiker, Phys. Rev. B 46, 12485 (1992).

${ }^{25}$ For a review, see M. Büttiker, Reversing the sign of current-current correlations, in "Quantum Noise", edited by Yu. V. Nazarov and Ya. M. Blanter (Kluwer, 2003).

26 D.A. Bagrets and Yu.V. Nazarov, Phys. Rev. B 67, 085316 (2003).

27 A. Cottet, W. Belzig and C. Bruder, Phys. Rev. Lett. 92, 206801 (2004); A. Cottet and W. Belzig, Europhys. Lett. 66, 405 (2004).

28 J. Börlin W. Belzig, and C. Bruder, Phys. Rev. Lett. 88, 197001 (2002).

29 P. Samuelsson and M. Büttiker, Phys. Rev. Lett. 89, 046601 (2002); Phys. Rev. B 66, 201306 (2002).

30 D. Sánchez, R. López, P. Samuelsson, and M. Büttiker Phys. Rev. B 68, 214501 (2003) .

31 G. Burkard, D. Loss, and E.V. Sukhorukov, Phys. Rev. B 61, R16 303 (2000).

32 P. Coleman, Phys. Rev. B 29, 3035 (1984).

33 There are other expressions in the literature to deal with asymmetries in the frequency dependence of $S$. Here we investigate the zero-frequency limit of $S$, for which all of them are equivalent. For recent works on symmetrized noise versus nonsymetrized noise, see R. Aguado and L.P. Kouwenhoven, Phys. Rev. Lett. 84, 1986 (2000); H.-A. Engel and D. Loss cond-mat/0312107 (preprint).

34 D.C. Langreth, in Linear and Nonlinear Electron Transport in Solids (J.T. Devreese and V.E. Van Doren, eds.), NATO 
ASI, Ser. B, Vol. 17 (Plenum, New York, 1976).

${ }^{35}$ For a textbook treatment, see H. Haug and A.P. Jauho, Quantum Kinetics in Transport and Optics of Semiconductors, Springer Series in Solid-State Sciences, SpringerVerlag, Berlin (1998).

36 M. Büttiker, Phys. Rev. Lett. 57, 1761 (1986).

37 R. Aguado and D.C. Langreth, Phys. Rev. Lett. 85, 1946 (2000).

38 Y.D. Wei, B.G. Wang, J. Wang, and H. Guo, Phys. Rev. B 60, 16900 (1999).

39 D.S. Fisher and P.A. Lee, Phys. Rev. B 23, 6851 (1981); H.U. Baranger and A.D. Stone, ibid. 40, 8169 (1989).

40 F.M. Souza, J.C. Egues, and A.P. Jauho, cond-mat/0209263 (unpublished).

41 J.-X. Zhu and A.V. Balatsky, Phys. Rev. B 67, 165326 (2003).

42 R. Lü and Z-R. Liu, cond-mat/0210350 (unpublished).

43 For a review, see D.M. Newns and N. Read, Adv. Phys. 36, 799 (1987).

44 M. Büttiker, Phys. Rev. Lett. 65, 2901 (1990).

45 Since we take the limit $U \rightarrow \infty$, we can safely neglect screening effects (the charge is fixed). However, in a realistic situation one should take into account a screening potential, which may become important in the nonlinear regime.

46 M. Büttiker, Phys. Rev. B 33, 3020 (1986).

47 Dephasing in a quantum dot in the Kondo regime by a quantum point contact has been treated theoretically in A. Silva and S. Levit, Europhys. Lett. 62, 103 (2003) and experimentally in M. Avinun-Kalish, M. Heiblum, A. Silva, D. Mahalu, and V. Umansky, Phys. Rev. Lett. 92, 156801 (2004).

48 The inelastic case for a Breit-Wigner resonance is treated by M. Büttiker, IBM J. Res. Developm. 32, 63 (1988).

49 M.J.M. de Jong and C.W.J. Beenakker, Physica A 230, 219 (1996).
50 Y. Meir and N.S. Wingreen, Phys. Rev. Lett. 68, 2512 (1992).

51 S.Y. Cho, H.-Q. Zhou, and R.H. McKenzie, Phys. Rev. B 68, 125327 (2003).

52 L.Y. Chen and C.S. Ting, Phys. Rev. B 43, R4534 (1991).

53 Semiconductor Spintronics and Quantum Computation, edited by D.D. Awschalom, D. Loss, and N. Samarth (Springer, Berlin, 2002).

54 N. Sergueev, Q.-f. Sun, H. Guo, B.G. Wang, and J. Wang, Phys. Rev. B 65, 165303 (2002).

55 P. Zhang, Q.-K. Xue, Y. Wang, and X.C. Xie, Phys. Rev. Lett. 89, 286803 (2002).

56 J. Martinek, M. Sindel, L. Borda, J. Barnaś, J. König, G. Schön, and J. von Delft Phys. Rev. Lett. 91, 247202 (2003).

57 M.-S. Choi, D. Sánchez and R. López, Phys. Rev. Lett. 92, 056601 (2004)

58 This is associated with the asymmetric Anderson model.

59 A.N. Pasupathy, R.C. Bialczak, J. Martinek, J.E. Grose, L.A.K. Donev, P.L. MacEuen, and D.C. Ralph, Science 306, 86 (2004).

60 J. Nygard, W.F. Koehl, N. Manson, L. DiCarlo, and C.M. Marcus, cond-mat/0410467 (unpublished).

61 R. López, R. Aguado, G. Platero, and C. Tejedor, Phys. Rev. B 64, 075319 (2001).

62 M. Henny, S. Oberholzer, C. Strunk, T. Heinzel, K. Ensslin, M. Holland, C. Schönenberger, Science 284, 296 (1999); W.D. Oliver, J. Kim, R.C. Liu, and Y. Yamamoto, ibid. 299 (1999).

63 D. Sánchez et al., in preparation (2004).

64 J. Park, A.N. Pasupathy, J.I. Goldsmith, C. Chang, Y. Yaish, J.R. Petta, M. Rinkoski, J.P. Sethna, H. D. Abruña, P.L. McEuen, D.C. Ralph, Nature (London) 417, 722 (2002); W. Liang, M.P. Shores, M. Bockrath, J.R. Lond, and H. Park, ibid. 417, 725 (2002). 